\title{
CORRECTIONS
}

\section{From promises to policies: is big pharma delivering on transparency?}

In this Feature by Peter Doshi (BMJ 2014;348:g1615, doi:10. 1136/bmj.g1615), Box 1 (The Chief Medical Officers

Cite this as: BMJ 2014;348:g2010

Roundtable (CMOR) are from 21 companies) only contains the

๑) BMJ Publishing Group Ltd 2014

names of 20 companies. Takeda is missing. 